\title{
A COUNTEREXAMPLE ON RELATIVE REGULAR NEIGHBORHOODS
}

\author{
BY RALPH TINDELL ${ }^{1}$
}

Communicated by J. Milnor, April 12, 1966

Hudson and Zeeman defined the concept of a relative regular neighborhood in [1], and gave an existence theorem and two uniqueness theorems; the purpose of this note is to show that the uniqueness theorems are false. A corrected version of these theorems has been announced by L. S. Husch and will appear later. For 3-manifolds, however, the corrected version is equivalent to the original.

For general terminology and definitions, see Zeeman [2]. Suppose $K, L$ are subcomplexes of some complex $J$. We say that $K$ is link collapsible on $L$ if $l k(A, \mathrm{Cl}(K-L))$ is collapsible for any simplex $A$ of $\mathrm{Cl}(K-L) \cap L$. If $X$ and $Y$ are compact polyhedra in a polyhedral manifold $M$, we say that $X$ is link collapsible on $Y$ if there is a triangulation $K, L$ of $X, Y$ such that $K$ is link collapsible on $L$. For example, it is easy to see that a manifold is always link collapsible on any subpolyhedron of its boundary. Let $X, Y, N$ be compact polyhedra in $M$. We say that $N$ is a regular neighborhood of $X \bmod Y$ in $M$ if

(1) $N$ is an $m$-manifold $(m=\operatorname{dim} M)$,

(2) $N$ is a topological neighborhood of $X-Y$ in $M$ and

$$
N \cap Y=N \cap Y=\mathrm{Cl}(X-Y) \cap Y,
$$

(3) $N$ collapses to $\mathrm{Cl}(X-Y)$.

The uniqueness theorems given by Hudson and Zeeman say, among other things, that any two regular neighborhoods of $X \bmod Y$ in $M$ are homeomorphic keeping $\mathrm{Cl}(X-Y)$ fixed, provided $X$ is link collapsible on $Y$.

Let $\left(B^{3}, B^{1}\right)$ be a knotted 3, 1 ball pair in $E^{3}$ and let $B^{4}=a * B^{3}$ and $B^{2}=a * B^{1}$ where $a=(0,0,0,1) \in E^{4}$ and $*$ denotes join. The 4,2 ball pair $\left(B^{4}, B^{2}\right)$ is locally knotted at the vertex $a$ and hence is knotted. However it is easy to see that $B^{2}$ is unknotted in $E^{4}$. Let $h: E^{4} \rightarrow E^{4}$ be an onto piecewise linear homeomorphism such that $h\left(B^{2}\right)=\Delta$ is a 2 -simplex. $B^{4}$ collapses cone-wise to $B^{2}$, so that $h\left(B^{4}\right)$ collapses to $h\left(B^{2}\right)=\Delta$. Also $\dot{\Delta} C h\left(\dot{B}^{4}\right)$ and $\stackrel{\Delta}{ } \subset\left(\stackrel{\circ}{B}^{4}\right)$, so that $h\left(B^{4}\right)$ is a regular neighborhood of $\Delta \bmod \dot{\Delta}$ in $E^{4}$. Let $\Sigma$ be the 2-fold suspension of $\Delta$ in $E^{4} ;$ then $\Sigma$ is a regular neighborhood of $\Delta \bmod \dot{\Delta}$ in $E^{4}$.

\footnotetext{
1 The author was supported by the National Science Foundation, Grant GP 4006
} 
If the above uniqueness theorem were true, there would be a homeomorphism carrying $h\left(\left(B^{4}\right), \Delta\right)$ onto $(\Sigma, \Delta)$ which implies that $\left(h\left(B^{4}\right), \Delta\right)$ $=\left(h\left(B^{4}\right), h\left(B^{2}\right)\right)$ is an unknotted ball pair. This is clearly false since it is a homeomorph of the knotted ball pair $\left(B^{4}, B^{2}\right)$. Clearly this type of argument can be carried out in $E^{n}$ for every $n \geqq 4$, so that the uniqueness theorem is false for all dimensions greater than 3; as remarked above, the theorem is true for 3-manifolds.

The author wishes to thank Professor Tatsuo Homma for suggesting a simplification of the author's counter-example.

\section{REFERENCES}

1. J. F. P. Hudson and E. C. Zeeman, On regular neighborhoods, Proc. London Math. Soc. (3) 11 (1964), 719-745.

2. E. C. Zeeman, Seminar on combinatorial topology, Inst. Hautes Etudes Sci. Publ. Math., Paris, 1963. (mimeographed notes)

3. L. S. Husch, On relative regular neighborhoods. Preliminary report, Abstract 66T-212, Notices Amer. Math. Soc. 13 (1966), 386.

Florida State University 